\title{
IMPACTO DA MORFOLOGIA DE PARQUE URBANO NO MICROCLIMA E NO CONFORTO TÉRMICO DE CUIABÁ - BRASIL
}

\author{
JUSTI, Ana Clara Alves - clarasevla@gmail.com \\ Universidade Federal do Mato Grosso / UFMT
}

\begin{abstract}
NOGUEIRA, Marta Cristina de Jesus Albuquerque - mcjanp@gmail.com
Universidade Federal do Mato Grosso / UFMT
\end{abstract}

\author{
SANTOS, Flávia Maria de Moura - flavia_mms@hotmail.com \\ Universidade Federal do Mato Grosso / UFMT
MUSIS, Carlos Ralph de Musis - carlo.demusis@gmail.com
Universidade de Cuiabá / UNIC

NOGUEIRA, José de Souza - nogueira@ufmt.br
Universidade Federal do Mato Grosso / UFMT

\begin{abstract}
RESUMO: A crescente urbanização promove a degradação contínua do ambiente urbano, como por exemplo, o acréscimo de revestimentos impermeáveis, a redução de áreas verdes, as alterações do albedo e da rugosidade superficial, a canalização de corpos hídricos e a emissão de poluentes por parte das indústrias, que de modo geral, modificam negativamente o microclima, o conforto térmico e consequentemente a qualidade de vida da sociedade. Nessa perspectiva, estudos revelam que os parques urbanos têm o potencial de mitigar alguns dos efeitos nocivos da urbanização. Portanto, objetivou-se com o presente estudo, verificar o microclima relativo às condições termo higrométricas de um parque, em comparação com uma área urbana de referência, avaliando suas contribuições no conforto térmico dos cidadãos. Para as variáveis temperatura do ar e umidade relativa do ar aferidas no parque, houve redução de até $5,2^{\circ} \mathrm{C}$ e aumento de até $10 \%$ quando comparadas à estação fixa em área urbana. Em relação ao conforto térmico, o parque variou a escala de percepção de conforto a desconforto e a estação fixa variou de pouco desconforto a desconforto. Assim, concluise que a morfologia do parque analisado contribui para que as condições termo higrométricas interfiram positivamente no microclima e no conforto térmico.
\end{abstract}

PALAVRAS-CHAVES: temperatura do ar, umidade relativa do ar, vegetação

MPACT OF URBAN PARK MORPHOLOGY IN MICROCLIMA AND THERMAL COMFORT IN CUIABÁ - BRAZIL

ABSTRACT: Growing urbanization promotes the continuous degradation of the urban environment, such as the addition of impermeable coatings, the reduction of green areas, changes in albedo and surface roughness, the channeling of water bodies and the emission of pollutants by industries, which in general, modify negatively the microclimate, the thermal comfort and consequently the quality of life of the society. From this perspective, studies show that urban parks have the potential to mitigate some of the harmful effects of urbanization. Therefore, the objective of this study was check the microclimate relative to the thermo hygrometric conditions of a park, in comparison with an reference urban area, evaluating its contributions in the thermal comfort of the citizens. For the variables air temperature and air relative humidity measured in the park, there was a reduction of up to $5.2^{\circ} \mathrm{C}$ and an increase of up to $10 \%$ when compared to the fixed station in an urban area. Regarding thermal comfort, the park ranged comfort perception scale to discomfort, and the fixed season varied from little discomfort to discomfort. Thus, it is concluded that the morphology of the park analyzed contributes to the that the thermo hygrometric conditions interfere positively in the microclimate and the thermal comfort. 
KEYWORDS: air temperature; relative humidity, vegetation.

\section{INTRODUÇÃO}

Mais da metade da população mundial habita áreas urbanas e estimativas sugerem que este número deverá atingir 66\% até 2050 (UNITED NATIONS, 2014), induzindo o crescimento citadino tanto em tamanho quanto em densidade e favorecendo o desenvolvimento econômico, social e cultural.

Entretanto, a rápida urbanização traz consigo não apenas estilos de vida modernos e convenientes em termos de centralização funcional, mas também a deterioração contínua do ambiente (SUN et al., 2017), como por exemplo, o acréscimo de revestimentos impermeáveis, a redução de áreas verdes, as alterações do albedo e da rugosidade superficial, a canalização de corpos hídricos e a emissão de poluentes por parte das indústrias, que de modo geral, contribuem para a ocorrência da ilha de calor urbano (UHI), interferindo negativamente no clima e consequentemente na qualidade de vida da sociedade (CHU et al., 2017).

A UHI é o fenômeno decorrente do aumento da temperatura do ar nas cidades em comparação com os ambientes rurais, caracterizando-se como uma das mudanças climáticas antropogênicas mais evidentes (NASTRAN et al., 2018).

Nessa perspectiva, consideráveis pesquisas foram realizadas, almejando compreender as peculiaridades de tal fenômeno e registrar a sua magnitude (OKE, 1982; ARNFIELD, 2003; SCHWARZ N, 2011; KOTHARKAR et al., 2018).

Dessa forma, os resultados dos estudos indicam que a intensidade da UHI pode atingir a diferença de temperatura do ar de $10 \mathrm{~K}$ dependendo das propriedades urbanas e das condições climáticas locais (OKE, 1973; MIHALAKAKOU et al., 1998), impactando diretamente na vida dos cidadãos em relação à intensificação das concentrações de poluentes atmosféricos e da piora das condições de conforto térmico (AKBARI et al., 1997; ROSSI, 2012; LIMA, 2016; MARRA, 2017 e QUERINO, 2017), além do significativo consumo de energia para o resfriamento das edificações (CARTALIS et al., 2001; KOLOKOTRONI et al., 2006; MIRZAEI \& HAGHIGHAT, 2010).

A ilha de calor urbano na cidade de Cuiabá - Brasil, ainda é pouco documentada, (XAVIER et al., 2009; FRANCO \& NOGUEIRA 2012; SANTOS, 2013; FRANCO et al., 2013), o que justifica a necessidade de trabalhos na área, uma vez que a temperatura média mensal do ar naturalmente varia entre $21,9^{\circ} \mathrm{C}$ e $31,3^{\circ} \mathrm{C}$ (MAITELLI, 1994) podendo agravar se o uso e a ocupação do solo não acontecer de modo apropriado.

Diversas técnicas de mitigação foram recomendadas para amenizar o impacto da UHI. Os mais difundidos e eficientes incluem o uso de materiais reflexivos em edifícios e estruturas urbanas (AKBARI \& LEVINSON, 2008; ZHANG et al., 2017) a inclusão de materiais com tecnologia avançada (KARLESSI et al., 2011; XAMAN et al., 2017), a incorporação de resfriamento terrestre e evaporativo (MIHALAKAKOU et al., 1994; ZHANG et al., 2017), o aumento da vegetação sob a forma de telhados verdes (SANTAMOURIS et al., 2012; BESIR, 2018) e a construção de parques urbanos e outras áreas arborizadas (SKOULIKA et al. 2014; LIN et al., 2015; ALGECIRAS et al., 2016). 
Os parques urbanos contribuem substancialmente para a melhoria do clima dos grandes centros e para o controle da UHI, pois contém espécies arbóreas que fornecem proteção e absorvem a radiação solar, influenciam os movimentos gasosos e trocas de calor, refrigeram o ar por meio dos processos de evapotranspiração. Além disso, as árvores filtram os poluentes atmosféricos, evitam erosões, diminuem os ruídos e proporcionam bem estar à aqueles que o utilizam (SKOULIKA et al. 2014).

No entanto, apesar de muitos trabalhos reportarem na literatura sobre os benefícios desencadeados pelos parques urbanos, poucos relatam os impactos da morfologia dos mesmos no microclima e no conforto térmico, principalmente em cidades do interior do Brasil, como o caso de Cuiabá.

Assim, o objetivo do presente estudo foi verificar o microclima relativo às condições termo higrométricas de um parque, em comparação com uma área urbana de referência, para os períodos da manhã e da tarde, nos intervalos de estiagem e chuvoso, avaliando suas contribuições no conforto térmico dos cidadãos.

\section{MATERIAIS E MÉTODOS}

\section{1 ÁREA DE ESTUDO}

A cidade de Cuiabá, capital do estado de Mato Grosso, está situada na região centro oeste brasileira, à $15^{\circ} 38^{\prime}$ de latitude sul e $56^{\circ} 06^{\prime}$ de longitude oeste, com altitude média inferior a $200 \mathrm{~m}$ acima do nível do mar. Segundo a classificação de Köppen-Geiger, o perfil climático cuiabano é tropical continental semiúmido do tipo $A w$, com duas estações representadas por chuvas na primavera-verão e por estiagem no outono-inverno.

A temperatura média máxima anual do ar em Cuiabá varia de $31^{\circ} \mathrm{C}$ a $34^{\circ} \mathrm{C}$ e a média mínima anual, varia entre 18 a $21^{\circ} \mathrm{C}$. Enquanto que a umidade relativa do ar tem a média anual de $70 \%$ no intervalo chuvoso, alcançando valores de $12 \%$ no intervalo de seca (MAITELLI, 2005).

O parque selecionado para a avaliação, foi o Parque Estadual Zé Bolo Flô, localizado na região sul da malha urbana de Cuiabá à $15^{\circ} 38^{\prime}$ de latitude sul e $56^{\circ} 03^{\prime}$ de longitude oeste, que possui uma área equivalente a $410.000 \mathrm{~m}^{2} \mathrm{de}$ unidade de conservação ambiental, sendo que dentro de seus limites estão inseridos a Escola de Saúde Pública Doutor Agrícola Paes de Barros, o Núcleo de Ofiologia, o Hospital Adauto Botelho, além de pistas e trilhas de caminhada em seu interior, (Figura 1). 


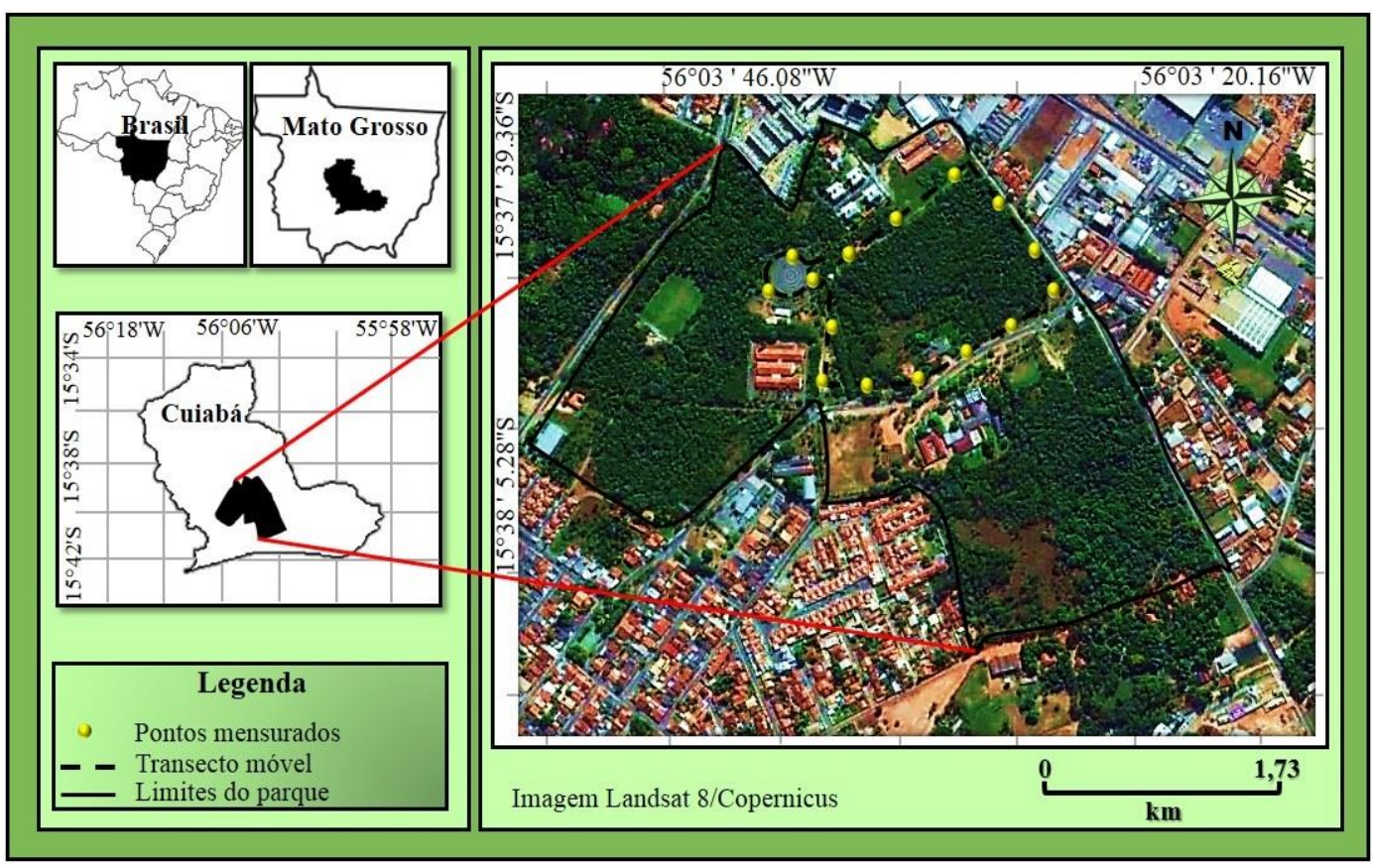

Figura 1 - Localização do Parque Zé Bolo Flô. Fonte: Adaptada de Google Earth, 2018

Conforme a Secretaria de Estado do Meio Ambiente de Mato Grosso (2012), o Parque Estadual Zé Bolo Flô tem a Floresta de Galeria como a fitofisionomia mais representativa em área, o relevo majoritariamente plano é caracterizado por entalhes fluviais de pequena profundidade, com predominância de declividades inferiores a $5 \%$. Além disso, no contexto da bacia do rio Cuiabá, o parque está inserido na bacia hidrográfica do rio Coxipó, afluente direto do rio Cuiabá, distando aproximadamente $300 \mathrm{~m}$ de sua margem esquerda.

\subsection{PROCEDIMENTO EXPERIMENTAL}

A pesquisa exploratória ocorreu em dias com condições atmosféricas favoráveis, ou seja, ventos fracos, céu com ausência de nuvens e consequentemente sem a formação de precipitação pluviométrica (OKE, 1982).

Efetuou-se a mesma tanto para o período matutino, com início às $8 \mathrm{~h} 00 \mathrm{~min}$, quanto para o período vespertino, com início às $14 \mathrm{~h} 00 \mathrm{~min}$, nos meses de julho a outubro de 2016, representativos do intervalo de estiagem e de novembro de 2016 a fevereiro de 2017, representativos do intervalo chuvoso. Entretanto, por se tratar de um estudo que depende das condições ambientais externas, foram amostrados apenas três dias consecutivos de cada mês, totalizando vinte e quatro medições para ambos os períodos.

O transecto móvel foi dividido em 15 pontos com características distintas entre si (Figura 2), representativos da totalidade do parque e que favoreciam a execução de todo o percurso em um prazo máximo de 1 h00min. 


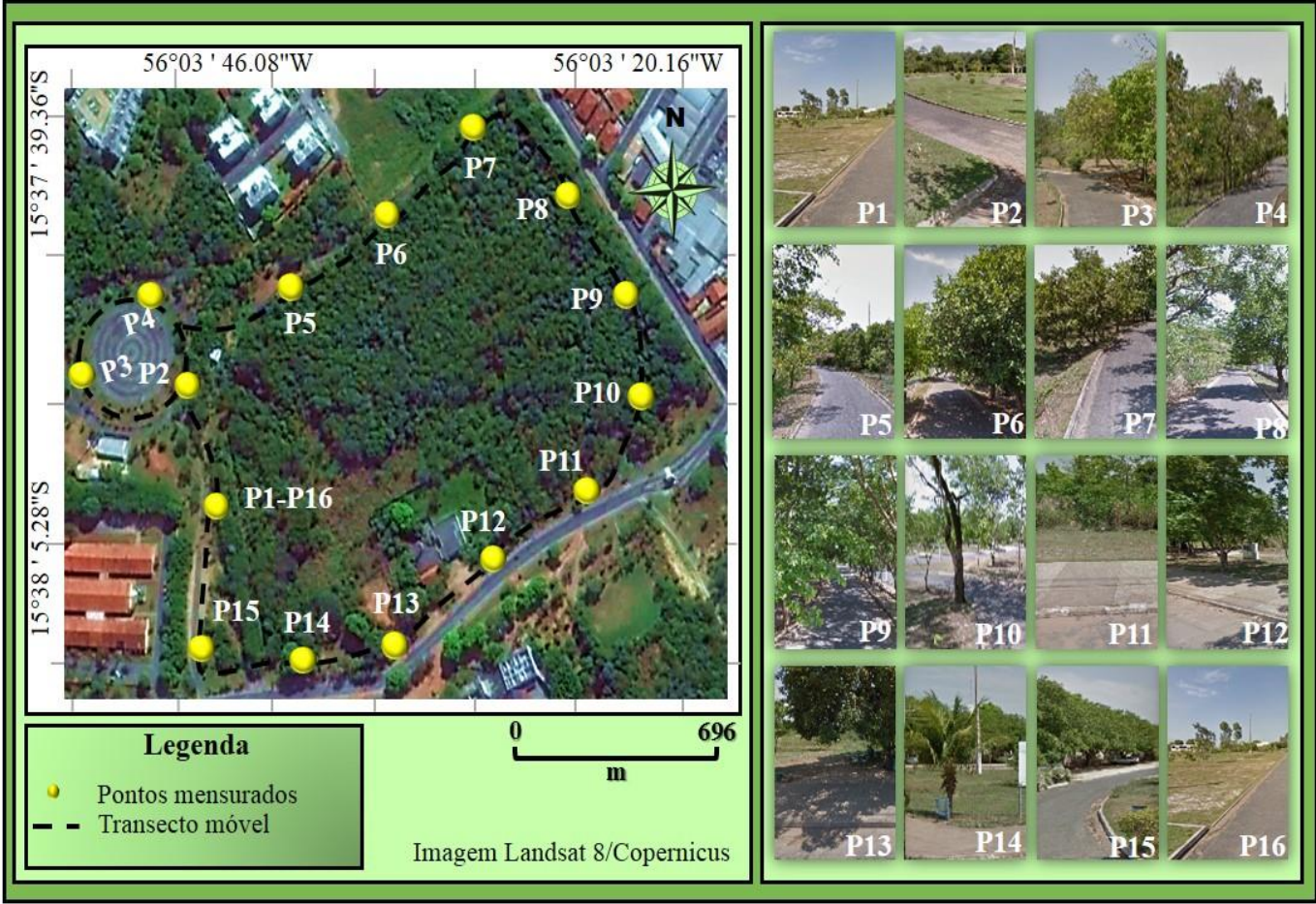

Figura 2 - Transecto móvel e os respectivos pontos amostrados.Fonte: Adaptado de Google Earth, 2018

Para não ultrapassar o prazo de 1h00min, a distância entre um ponto e o outro foi percorrida em aproximadamente 1 min30s, com uma parada de aproximadamente 2 min em cada ponto para a estabilização do equipamento.

Assim, a temperatura do ar $\left(\mathrm{T}^{\circ} \mathrm{C}\right)$ bem como a umidade relativa do ar (UR\%) no interior do parque, foram registradas por intermédio de um sensor termo- higrômetro data logger, modelo HOBO - Onset, protegido por um abrigo alternativo de Policloreto de Vinila (PVC), (Figura 3).

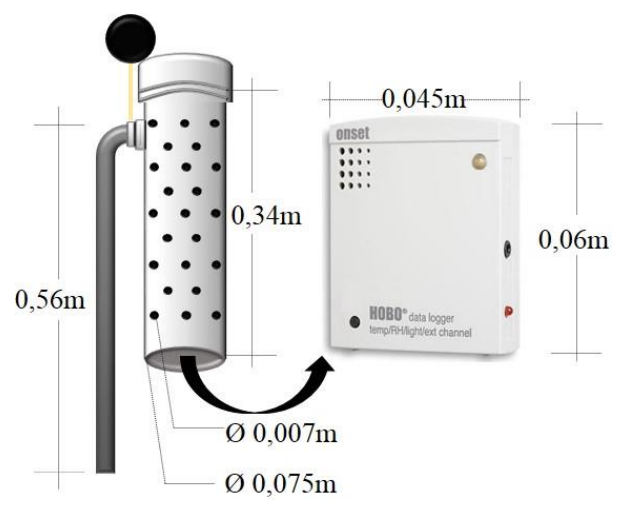

Figura 3 - Esquema representativo do abrigo alternativo de PVC e sensor termo higrômetro 
É válido ressaltar que apesar de ser um abrigo fora dos padrões normativos, o mesmo foi construído por pesquisadores do clima e mostrou-se adequado para a coleta de dados em estudos que usam a metodologia de transecto (SANTOS, 2012; VALIN JÚNIOR et al., 2016; De PAULA, 2017 e QUERINO, 2017). Além disso, diante de recursos limitados e dependentes de editais, contornar essas dificuldades com soluções viáveis economicamente, torna-se cada vez mais necessário.

A precisão do sensor de $\left(T^{\circ} \mathrm{C}\right)$ foi de $1^{\circ} \mathrm{C}$ operando em um intervalo de -20 a $50^{\circ} \mathrm{C}$ e a precisão do sensor de (UR\%) foi de $\pm 3 \%$ operando em um intervalo de 0 a $100 \%$. No mais, a frequência de amostragem foi de 5 segundos e posicionou-se o mesmo a $1,10 \mathrm{~m}$ acima da superfície do solo, conforme a norma ISO 7726 (ISO, 1998).

Já a temperatura do ar e a umidade relativa do ar da região urbanizada, foi aferida através de uma estação meteorológica fixa modelo HOBO U30-NRC Onset, pertencente ao Programa de Pós-Graduação em Física Ambiental, localizada dentro da Universidade Federal de Mato Grosso (UFMT) à $15^{\circ} 36^{\prime}$ de latitude sul e $56^{\circ} 03^{\prime}$ de longitude oeste, (Figura 4).

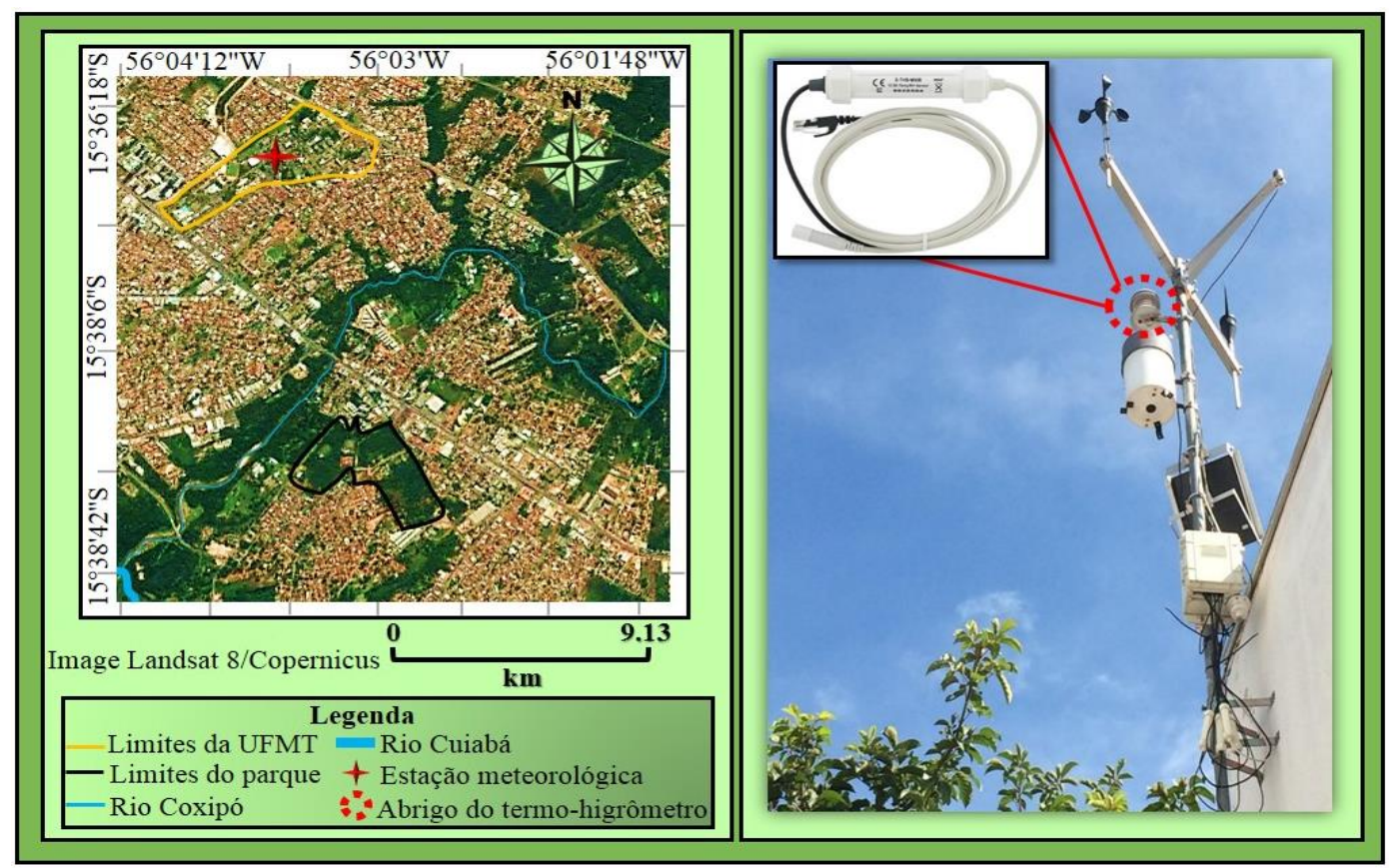

Figura 4 - Localização da estação microclimática fixa e sua respectiva estrutura. Fonte: Adaptada de Google Earth, 2018

Utilizou-se os dados do sensor de temperatura e umidade S-THB-M002 Onset, com precisão de $\pm 0,21^{\circ} \mathrm{C}$ e intervalo de operação de $-40^{\circ}$ a $75^{\circ} \mathrm{C}$ para a $\left(\mathrm{T}^{\circ} \mathrm{C}\right)$ e com precisão de $\pm 2,5 \%$ e intervalo de operação de 0 a $100 \%$ para a (UR\%). No mais, a frequência de amostragem também foi de 5 segundos e a estação foi posicionada à $\cong 6,85 \mathrm{~m}$ do nível do solo, livre de interferência arbórea e de edificações em seu entorno.

É importante frisar que os sensores são verificados (ASTM, norma: D443000) e calibrados (ISO, norma: 17025/17) periodicamente, com a finalidade de 
controlar o desempenho dos mesmos e manter a comparabilidade operacional da coleta de dados entre eles.

\subsection{CÁLCULO DE CONFORTO TÉRMICO}

Fez-se o uso do método proposto por Thom (1959), denominado de Índice de Temperatura e Umidade (ITU). Este índice é um estimador de conforto térmico humano, baseado em condições de temperatura do ar e umidade relativa do ar, considerando as trocas de calor por convecção livre.

A escolha desse índice em específico, baseou-se na aplicação diante dos dados disponíveis, já que pelo horário de desenvolvimento do estudo, a quantidade de questionários aplicados aos usuários do parque e da região densamente construída foram insuficientes, impossibilitando a utilização de outros tipos de índices.

No entanto, trata-se de um ótimo avaliador de conforto térmico, aplicado em diversos ramos, dentre eles, o industrial, o agropecuário, ambientes de trabalho, supermercados e espaços abertos.

Assim, o mesmo é dado pela Equação:

$$
I T U=T-0,55(1-U R)(T-14)
$$

\section{Sendo:}

$\mathrm{T}$ : A temperatura do ar dada em ${ }^{\circ} \mathrm{C}$;

UR: A umidade relativa do ar dada em fração decimal.

Posteriormente, os resultados obtidos foram comparados com os valores de referência, dispostos na Tabela 01 .

Tabela 1 - Índice de Temperatura-Umidade (ITU)

\begin{tabular}{cc}
\hline Faixa & Percepção \\
\hline$<15^{\circ} \mathrm{C}$ & Frio \\
$15^{\circ} \mathrm{C}-20^{\circ} \mathrm{C}$ & Conforto \\
$20^{\circ} \mathrm{C}-25^{\circ} \mathrm{C}$ & Pouco desconforto \\
$>25^{\circ} \mathrm{C}$ & Desconforto \\
\hline
\end{tabular}

Fonte: Thom, 1959 (adaptada pelos autores)

De acordo com Thom (1959) os valores encontrados acima de $25^{\circ} \mathrm{C}$, são considerados pela maioria como desconfortável, enquanto que os valores entre 15 e $20^{\circ} \mathrm{C}$ são aceitos pela maioria como confortáveis. 


\subsection{ANÁLISE DE DADOS}

Posteriormente à organização dos dados de temperatura do ar, umidade relativa do ar e de conforto térmico em planilhas do Microsoft Excel, definiu-se o diagrama de caixas como ferramenta gráfica para representar a variação dos mesmos. $\mathrm{E}$, para critério de comparação, foi utilizada a análise de variância (ANOVA) acoplada ao teste de Tukey, a fim de verificar diferenças significativas, utilizando-se do software Sisvar versão 4.0.

\section{RESULTADOS E DISCUSSÃO}

\subsection{DISTRIBUIÇÃO DA TEMPERATURA DO AR E DA UMIDADE RELATIVA DO AR NO PARQUE}

A distribuição da temperatura do $\operatorname{ar}\left(\mathrm{T}^{\circ} \mathrm{C}\right)$ registrada no parque tanto para o intervalo de estiagem quanto para o intervalo chuvoso é apresentada na Figura 5-a) e b) respectivamente.
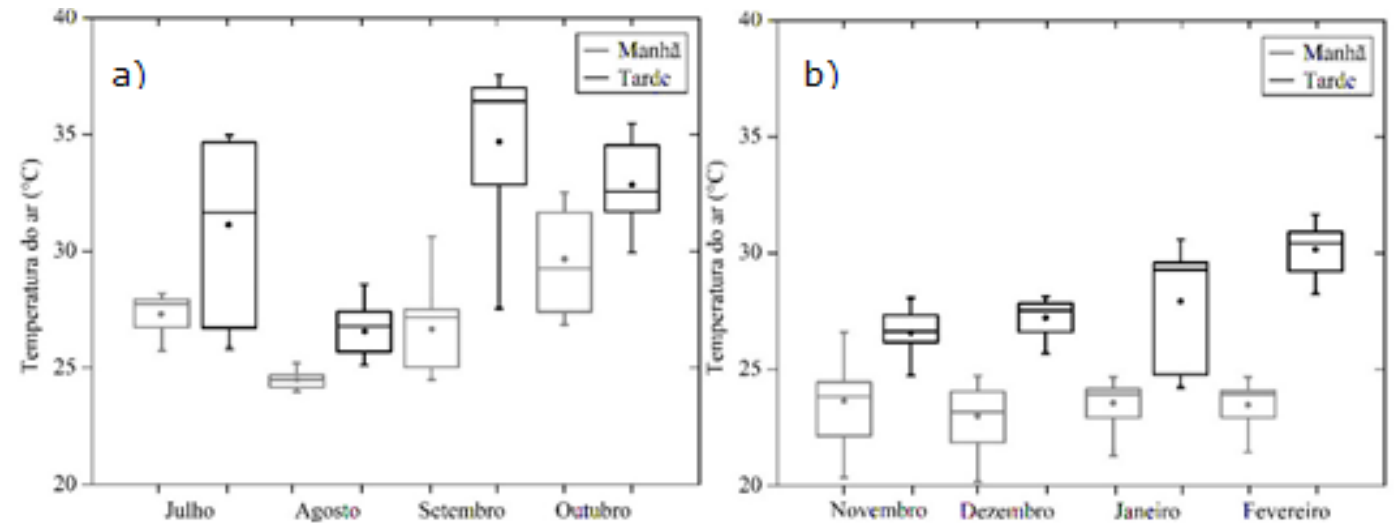

Figura 5 a) - Temperatura do ar no intervalo de estiagem e b) - temperatura do ar no intervalo chuvoso

As médias mínimas de $\left(\mathrm{T}^{\circ} \mathrm{C}\right)$ em ambos os intervalos, foram registradas às 8 h00 local, características do período da manhã e próximas ao nascer do sol. E as máximas, foram aferidas às $14 \mathrm{~h} 00$ local, características do período da tarde, quando o ângulo zenital apresenta seu menor tamanho e consequentemente a maior irradiância solar.

Para o intervalo de estiagem, os valores mínimos de $\left(\mathrm{T}^{\circ} \mathrm{C}\right)$ variaram de 23,9 a $26,8^{\circ} \mathrm{C}$ no período da manhã e de 25,1 a $30,0^{\circ} \mathrm{C}$ no período da tarde. Já os valores máximos de $\left(\mathrm{T}^{\circ} \mathrm{C}\right)$ variaram de 25,1 a $32,2^{\circ} \mathrm{C}$ e de 28,5 a $37,5^{\circ} \mathrm{C}$ no período manhã e da tarde respectivamente.

Em contrapartida, no intervalo chuvoso, os valores mínimos de $\left(\mathrm{T}^{\circ} \mathrm{C}\right)$ variaram de 20,1 a $22,1^{\circ} \mathrm{C}$ no período da manhã e de 24,2 a $28,2^{\circ} \mathrm{C}$ no período da tarde. Enquanto que os valores máximos de $\left(\mathrm{T}^{\circ} \mathrm{C}\right)$ variaram de 24,1 a $25,8^{\circ} \mathrm{C}$ e de 28,1 a $31,6^{\circ} \mathrm{C}$ no período da manhã e tarde respectivamente.

A maior amplitude térmica foi registrada para o intervalo de estiagem, com diferenças de 2,9 a $9,0^{\circ} \mathrm{C}$ em relação ao intervalo chuvoso, com diferenças de 1,7 a $3,5^{\circ} \mathrm{C}$. Estes resultados estão de acordo com os trabalhos de Barradas (1991), que relatou uma diferença de $\left(\mathrm{T}^{\circ} \mathrm{C}\right)$ de até $4^{\circ} \mathrm{C}$ para a cidade de México, 
com o de Petrali et al. (2009), que relataram diferenças de $\left(\mathrm{T}^{\circ} \mathrm{C}\right) \mathrm{de}$ aproximadamente $3,2^{\circ} \mathrm{C}$ entre as diferentes zonas do parque em Florença, Itália. Com o de Mahmoud (2011), que descobriu no Cairo, Egito, a diferença máxima de $\left(\mathrm{T}^{\circ} \mathrm{C}\right)$ entre as diferentes zonas de um parque era próxima de $4,7^{\circ} \mathrm{C}$ e, finalmente, com o de Skoulika et al. (2014), que apontaram diferenças de $\left(\mathrm{T}^{\circ} \mathrm{C}\right)$ mínimas de cerca de $9,5^{\circ} \mathrm{C}$ e de $\left(\mathrm{T}^{\circ} \mathrm{C}\right)$ máximas de aproximadamente $11,4^{\circ} \mathrm{C}$.

Sendo assim, as diferenças de temperatura do $\operatorname{ar}\left(\mathrm{T}^{\circ} \mathrm{C}\right)$ registradas no parque em estudo, podem ser atribuídas principalmente ao resfriamento radiativo, ao sombreamento arbóreo e seus respectivos processos de evapotranspiração, fatores estes que associados à morfologia do parque, contribuem para a distribuição das temperaturas ao longo de todo o trajeto percorrido.

Em paralelo, a distribuição de umidade relativa do ar (UR\%) para o intervalo de estiagem e chuvoso é apresentada na Figura 6-a) e b).
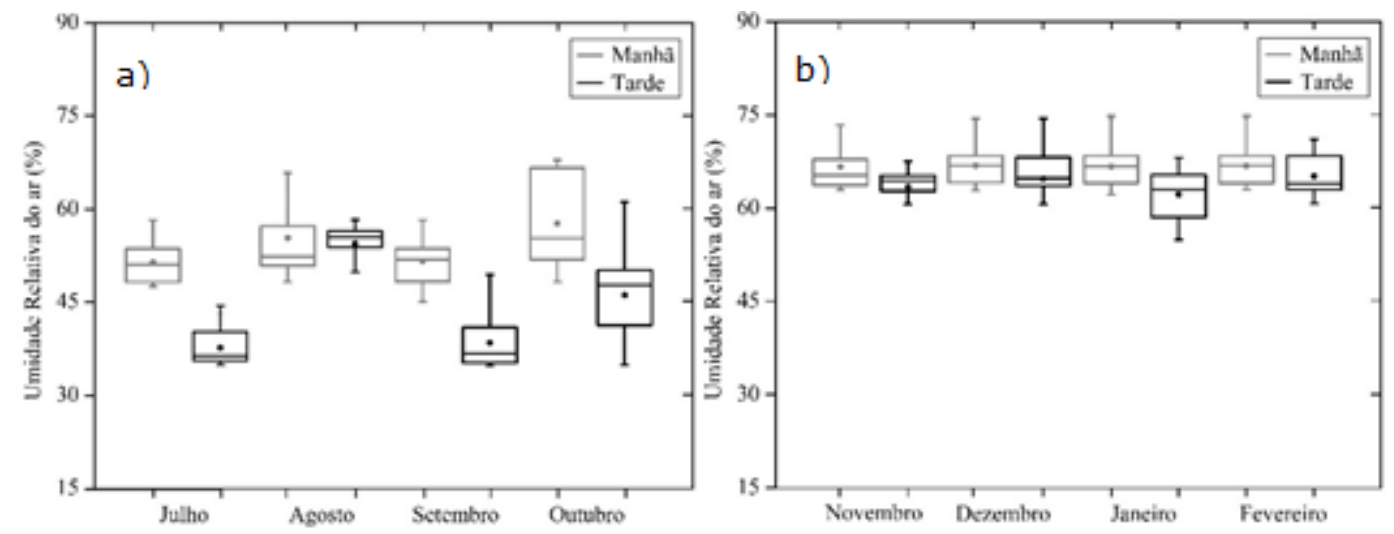

Figura 6 a) - Umidade relativa do ar no intervalo de estiagem e b) - umidade relativa do ar no intervalo chuvoso

Inversamente proporcional à distribuição das $\left(\mathrm{T}^{\circ} \mathrm{C}\right)$ e em ambos os intervalos, a (UR\%) registrou médias mínimas no período da tarde e médias máximas no período da manhã, fato que pode ser explicado de modo análogo ao comportamento da temperatura do ar.

Para o intervalo de estiagem, os valores mínimos de (UR\%) variaram de 35 a $47 \%$ no período da tarde e de 45 a $48 \%$ no período da manhã. Já os valores máximos de (UR\%) variaram de 44 a $61 \%$ e de 58 a $68 \%$ no período da tarde e manhã respectivamente.

Em contrapartida, no intervalo chuvoso, os valores mínimos de (UR\%) variaram de 62 a $67 \%$ no período da tarde e de 68 a $69 \%$ no período da manhã. Já os valores máximos de (UR\%) variaram de 73 a $78 \%$ e de no período da tarde e de 77 a $78 \%$ e manhã respectivamente.

A maior amplitude higrométrica também foi registrada para o intervalo de estiagem, com diferenças de 10 a 26\% em relação ao intervalo chuvoso, com diferenças de 8 a 15\%. Os resultados alcançados estão em conformidade com os trabalhos de Mahmoud (2011), que apresentou (UR\%) com diferenças de 2 a $12 \%$ entre as diferentes zonas de parque urbano de regiões quentes e áridas, 
com o de Skoulika et al. (2014) que registraram variação de (UR\%) de 5 a 35\% dentro dos limites do parque em Atenas e com o de Tong et al. (2017), que apresentaram uma diferença de (UR\%) de 10 a 15\% entre o verão e o inverno do norte da China.

Dessa forma, tendo em vista que a umidade relativa do ar (UR\%) varia de acordo com o comportamento das temperaturas do ar ao decorrer do dia (VAREJÃO, 2006), as diferenças de (UR\%) aferidas no parque de interesse, também podem ser atribuídas ao resfriamento radiativo durante a noite, ao sombreamento arbóreo e fundamentalmente aos processos de evapotranspiração, que em conjunto com a morfologia do parque, contribuem para o aumento ou a diminuição das (UR\%) durante o trajeto efetuado.

\subsection{DISTRIBUIÇÃO DA TEMPERATURA DO AR E DA UMIDADE RELATIVA DO AR NA ESTAÇÃO FIXA}

A distribuição de temperatura do $\operatorname{ar}\left(\mathrm{T}^{\circ} \mathrm{C}\right)$ na estação fixa urbana para o intervalo de estiagem e chuvoso é apresentada na Figura 7-a) e b).
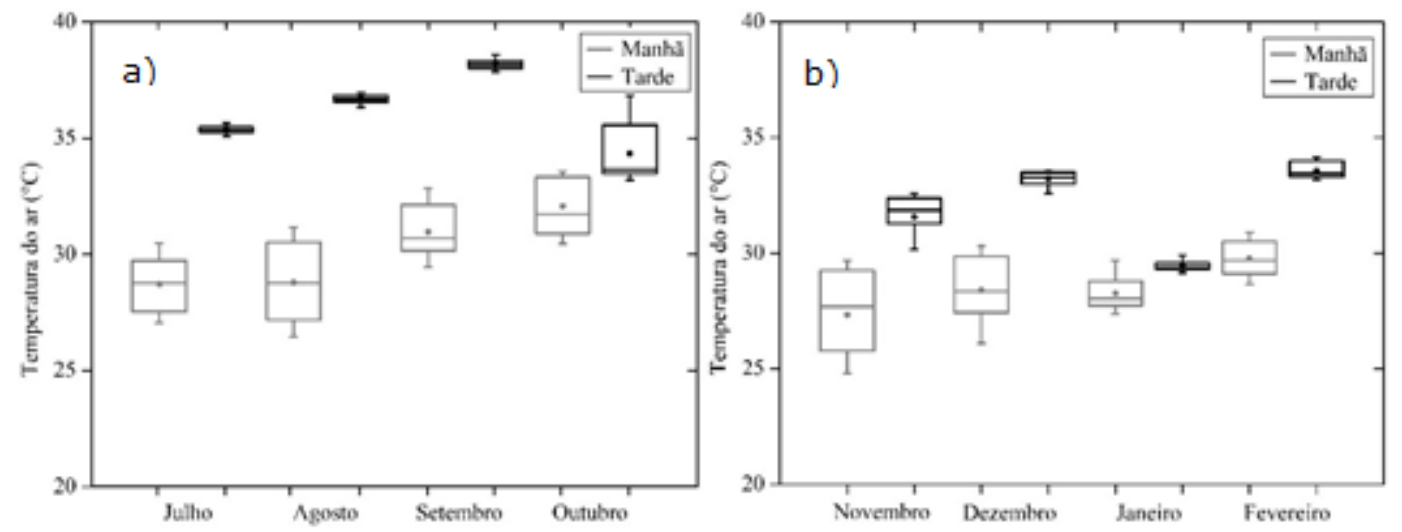

Figura 7 a) - Temperatura do ar no intervalo de estiagem e b) - temperatura do ar no intervalo chuvoso

Diferentemente das $\left(\mathrm{T}^{\circ} \mathrm{C}\right)$ registradas pelo método de transecto móvel, na estação fixa urbana, tanto o intervalo de estiagem, quanto o intervalo chuvoso obtiveram amplitude térmica similar, em que as diferenças foram de 0,8 a $5,0^{\circ} \mathrm{C}$.

O intervalo de estiagem, os valores mínimos de $\left(\mathrm{T}^{\circ} \mathrm{C}\right)$ variaram de 26,0 a $30,5^{\circ} \mathrm{C}$ no período da manhã e de 33,2 a $37,8^{\circ} \mathrm{C}$ no período da tarde. Já os valores máximos de $\left(\mathrm{T}^{\circ} \mathrm{C}\right)$ variaram de 30,5 a $32,9^{\circ} \mathrm{C}$ e de 35,7 a $38,6^{\circ} \mathrm{C}$ no período manhã e da tarde respectivamente.

Em contrapartida, no intervalo chuvoso, os valores mínimos de $\left(\mathrm{T}^{\circ} \mathrm{C}\right)$ variaram de 24,4 a $28,7^{\circ} \mathrm{C}$ no período da manhã e de 30,1 a $33,1^{\circ} \mathrm{C}$ no período da tarde. Enquanto que os valores máximos de $\left(\mathrm{T}^{\circ} \mathrm{C}\right)$ variaram de 29,4 a $30,9^{\circ} \mathrm{C}$ e de 30,0 a $34,2^{\circ} \mathrm{C}$ no período da manhã e tarde respectivamente.

Resultados semelhantes, podem ser verificados nos trabalhos de Nince (2013), cujas temperaturas médias diárias foram de 22 a $30^{\circ} \mathrm{C}$ e de 16 a $30^{\circ} \mathrm{C}$ para os intervalos de estiagem e chuvoso respectivamente e com o de Valin 
Júnior et al. (2015), cuja temperatura média diária para o período de estiagem foi de $27,7^{\circ} \mathrm{C}$.

Concomitantemente, a distribuição de umidade relativa do ar (UR\%) para o intervalo de estiagem e chuvoso é apresentada na Figura 8-a) e b).
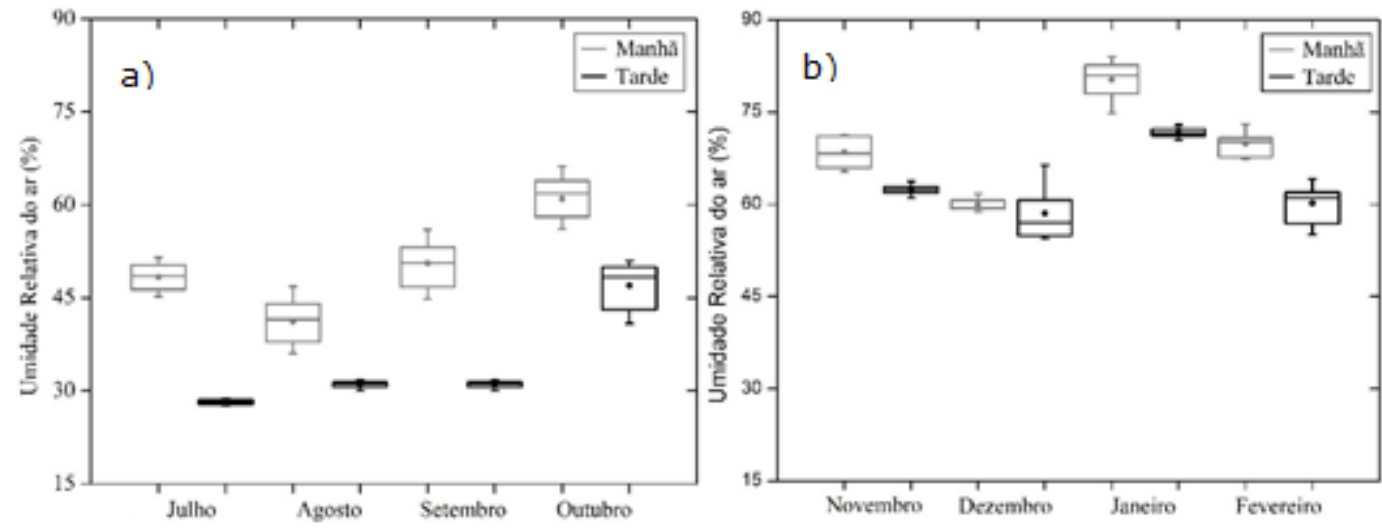

Figura 8 a) - Umidade relativa do ar no intervalo de estiagem e b) - umidade relativa do ar no intervalo chuvoso

Para o intervalo de estiagem, os valores mínimos de (UR\%) variaram de 28 a $41 \%$ no período da tarde e de 36 a $56 \%$ no período da manhã e os valores máximos variaram de 27 a $51 \%$ e de 47 a $66 \%$ no período da tarde e manhã respectivamente.

Em contrapartida, no intervalo chuvoso, os valores mínimos de (UR\%) variaram de 54 a $70 \%$ no período da tarde e de 59 a $75 \%$ no período da manhã e os valores máximos variaram de 64 a $73 \%$ e de 62 a $84 \%$ no período da tarde e manhã respectivamente.

Ao comparar os intervalos amostrados, a amplitude higrométrica no período da manhã foi maior para o intervalo de estiagem, com diferenças de 6 a $11 \%$ em relação ao chuvoso, com diferenças de 6 a $9 \%$ do intervalo chuvoso. Entretanto, no período da tarde, a maior amplitude higrométrica foi registrada para o intervalo chuvoso, com diferenças de 3 a $15 \%$ em comparação com ao de estiagem, com diferenças de 1 a $10 \%$.

Dessa forma, os resultados condizem com os trabalhos realizados também na cidade de Cuiabá, por Franco (2013), cujos valores de (UR\%) variaram de 2 a $11 \%$ e por De Paula (2017), cujas médias de (UR\%) ficaram entre 51 e 78\% para o intervalo seco e chuvoso respectivamente.

\subsection{COMPARAÇÃO DA TEMPERATURA DO AR E DA UMIDADE RELATIVA DO AR NO PARQUE E NA ESTAÇÃO FIXA}

A avaliação entre os tratamentos de intervalos (estiagem - chuvoso), períodos (manhã - tarde) e métodos (transecto no parque - estação fixa urbana), foi detalhada por Análise de Variância Univariada - ANOVA (F), detectando, em consonância, os efeitos estatisticamente significativos tanto para a temperatura do ar, quanto para a umidade relativa do ar, (Tabela 1 e 3 ). 
Tabela 1 - Análise de Variância para a variável temperatura do $\operatorname{ar}\left(\mathrm{T}^{\circ} \mathrm{C}\right)$

\begin{tabular}{cccccc}
\hline Tratamentos & GL & SQ & QM & FC & P-valor \\
\hline Intervalos & 1 & 21930.7 & 21930.7 & 3535.5 & $0.0000^{*}$ \\
Métodos & 1 & 4267.6 & 4267.6 & 688.0 & $0.0000^{*}$ \\
Períodos & 1 & 35640.8 & 35640.8 & 5745.8 & $0.0000^{*}$ \\
Intervalos x Métodos & 1 & 23.0 & 23.0 & 3.7 & $0.0540 *$ \\
Intervalos x Períodos & 1 & 45.1 & 45.1 & 7.3 & $0.0070^{*}$ \\
Métodos x Períodos & 1 & 3.5 & 3.5 & 0.6 & 0.4512 \\
Interv. x Períod. x Métod. & 1 & 123.7 & 123.7 & 19.9 & $0.0000^{*}$ \\
\hline
\end{tabular}

GL: Graus de liberdade, SQ: Soma dos quadrados, QM: Quadrado médio, FC: F calculado * Significativo ao nível de $5 \%$ de probabilidade

De acordo com o teste $\mathrm{F}$, para a variável temperatura do ar foi encontrado evidências de diferenças significativas ao nível de $5 \%$ de probabilidade, entre todos os tratamentos, com exceção para o tratamento de "métodos x períodos". Sendo assim, rejeita-se portanto a hipótese de nulidade $\mathrm{HO}$, ou seja, existe pelo menos um contraste significativo entre as médias de tratamento.

Nessa perspectiva, a fim de avaliar a magnitude das diferenças, foi utilizado o teste de Tuckey de comparações múltiplas, usando a interação tripla que contempla todos os tratamentos "intervalos x períodos x métodos", (Tabela 2).

Tabela 2 - Teste de Tuckey aplicado à interação tripla para a variável temperatura do ar $\left(\mathrm{T}^{\circ} \mathrm{C}\right)$

\begin{tabular}{|c|c|c|c|c|}
\hline & \multicolumn{2}{|c|}{ Transecto no parque } & \multicolumn{2}{|c|}{ Estação fixa urbana } \\
\hline & Estiagem & Chuvoso & Estiagem & Chuvoso \\
\hline Manhã & $27,0^{\circ} \mathrm{C}$ & $23,2^{\circ} \mathrm{C}$ & $30,1^{\circ} \mathrm{C}$ & $28,4^{\circ} \mathrm{C}$ \\
\hline Tarde & $31,3^{\circ} \mathrm{C}$ & $28,0^{\circ} \mathrm{C}$ & $36,1^{\circ} \mathrm{C}$ & $32,0^{\circ} \mathrm{C}$ \\
\hline
\end{tabular}

Observa-se com a Tabela 2 que os valores das médias de temperatura do ar $\left(\mathrm{T}^{\circ} \mathrm{C}\right)$ também se diferiram assim como os valores apresentados nos tópicos 3.1 e 3.2, em que a distribuição da $\left(\mathrm{T}^{\circ} \mathrm{C}\right)$ no parque apresentou-se menor quando comparada com a estação fixa em meio urbano.

As diferenças das médias de $\left(\mathrm{T}^{\circ} \mathrm{C}\right)$ aferidas pela manhã e pela tarde, no intervalo de estiagem, foram de 3,1 e $4,8^{\circ} \mathrm{C}$ respectivamente. Já as diferenças de $\left(\mathrm{T}^{\circ} \mathrm{C}\right)$ aferidas pela manhã e pela tarde, no intervalo de chuvoso, foram de 5,2 e $4,0^{\circ} \mathrm{C}$ respectivamente. Essas diferenças térmicas entre o parque e a área urbana podem ser justificadas pelas configurações urbanísticas presentes nas grandes cidades, em que a densidade de edificações e pavimentações, contribuem para a maior capacidade de absorção da radiação solar, emitindo consequentemente maior radiação na forma de calor, o que não ocorre semelhantemente em as áreas vegetadas.

Logo, fica evidente a importância da implementação de áreas verdes na tentativa de amenizar os impactos microclimáticos decorrentes dos avanços citadinos. Sendo assim, estudos trazem resultados semelhantes que reforçam 
essa necessidade, como por exemplo o de Valin Júnior et al. (2015), cuja diferença registrada foi de aproximadamente $2^{\circ} \mathrm{C}$ de temperatura média do ar.

Em paralelo, a Análise de Variância Univariada - ANOVA (F), feito para a variável umidade relativa do ar (UR\%), pode ser verificada na Tabela 3 a seguir.

Tabela 3 - Análise de Variância para a variável umidade relativa do ar (UR\%)

\begin{tabular}{cccccc}
\hline Grupos & GL & SQ & QM & FC & P-valor \\
\hline Intervalos & 1 & 789114.8 & 789114.8 & 26525.3 & $0.0000^{*}$ \\
Métodos & 1 & 6629.2 & 6629.2 & 222.8 & $0.0000^{*}$ \\
Períodos & 1 & 68050.9 & 68050.9 & 2287.4 & $0.000 *^{*}$ \\
Intervalos x Métodos & 1 & 503.2 & 503.2 & 16.9 & $0.0000^{*}$ \\
Intervalos x Períodos & 1 & 25123.7 & 25123.7 & 844.5 & $0.0000^{*}$ \\
Métodos x Períodos & 1 & 1525.6 & 1525.65 & 51.3 & 0.1624 \\
Interv. x Períod. x Métod. & 1 & 58.0 & 58.0 & 2.0 & $0.0000^{*}$ \\
\hline
\end{tabular}

GL: Graus de liberdade, SQ: Soma dos quadrados, QM: Quadrado médio, FC: F calculado.* Significativo ao nível de $5 \%$ de probabilidade

Similar à ANOVA (F), realizada para a variável temperatura do ar, conforme 0 teste $F$, para a (UR\%) também foi registrado evidências de diferenças significativas ao nível de $5 \%$ de probabilidade, entre todos os tratamentos, com exceção para o tratamento de "métodos x períodos", rejeitando-se portanto a hipótese de nulidade $\mathrm{HO}$, ou seja, existe pelo menos um contraste significativo entre as médias de tratamento.

Dessa forma, empregou-se o teste de Tuckey para avaliar a magnitude das diferenças das médias, usando a interação tripla que abrange todos os tratamentos, Tabela 4.

Tabela 4 - Teste de Tuckey aplicado à interação tripla para a variável umidade relativa do $\operatorname{ar}($ UR\%)

\begin{tabular}{ccccc}
\hline & \multicolumn{2}{c}{ Transecto no parque } & \multicolumn{2}{c}{ Estação fixa urbana } \\
& Estiagem & Chuvoso & Estiagem & Chuvoso \\
\hline Manhã & $54 \%$ & $71 \%$ & $50 \%$ & $70 \%$ \\
Tarde & $44 \%$ & $69 \%$ & $34 \%$ & $63 \%$ \\
\hline
\end{tabular}

As diferenças das médias de (UR\%) também se diferiram de modo igual ao que foi apresentado nos tópicos 3.1 e 3.2, consolidando a importância da contribuição de áreas verdes nas cidades construídas e em construção, sendo que para o intervalo de estiagem, pela manhã e pela tarde a diferença foi de $4 \mathrm{e}$ $10 \%$ respectivamente. Já para o intervalo chuvoso, pela manhã e pela tarde a diferença foi de 2 e $6 \%$ na devida ordem.

Visto que a (UR\%) é dependente da $\left(\mathrm{T}^{\circ} \mathrm{C}\right)$, as diferenças das médias registradas tanto no parque, quanto na estação fixa urbana é explicado de modo análogo, em que as maiores e menores $\left(\mathrm{T}^{\circ} \mathrm{C}\right)$ acarretam em menores e maiores 
(UR\%) mutuamente, o que também pode ser verificado no trabalho de Valin Júnior et al. (2015).

\subsection{ANÁLISE DE CONFORTO TÉRMICO}

A distribuição dos Índices de Temperatura e Umidade (ITU ${ }^{\circ} \mathrm{C}$ ) para 0 transecto realizado no parque é apresentada na Figura 9-a) e b).
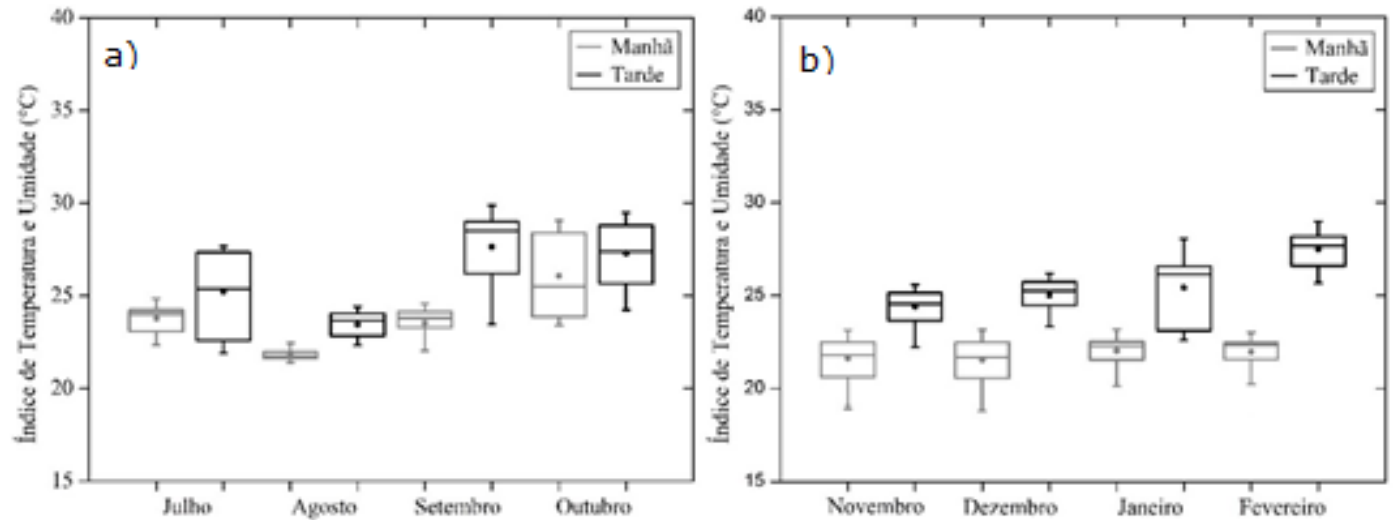

Figura 9 a) - Índice de Temperatura e Umidade no intervalo de estiagem b) - Índice de Temperatura e Umidade no intervalo chuvoso

Refletindo o comportamento das variáveis aferidas, os (ITU ${ }^{\circ}$ ) para o parque obteve menores valores no intervalo chuvoso e no período da manhã, quando comparado com o intervalo de estiagem e no período da tarde.

Para o intervalo chuvoso, os valores mínimos de $\left(\right.$ ITU $\left.^{\circ} \mathrm{C}\right)$ variaram de 18,6 a $19,0^{\circ} \mathrm{C}$ no período da manhã e de 22,2 a $25,7^{\circ} \mathrm{C}$ no período da tarde. Já os valores máximos de $\left(\mathrm{T}^{\circ} \mathrm{C}\right)$ variaram de 23,0 a $23,2^{\circ} \mathrm{C}$ e de 25,4 a $29,0^{\circ} \mathrm{C}$ no período manhã e da tarde respectivamente, variando a escala de percepção de conforto a desconforto.

A distribuição dos Índices de Temperatura e Umidade (ITU ${ }^{\circ} \mathrm{C}$ ) para a estação fixa em região urbana é apresentada na Figura 10-a) e b).
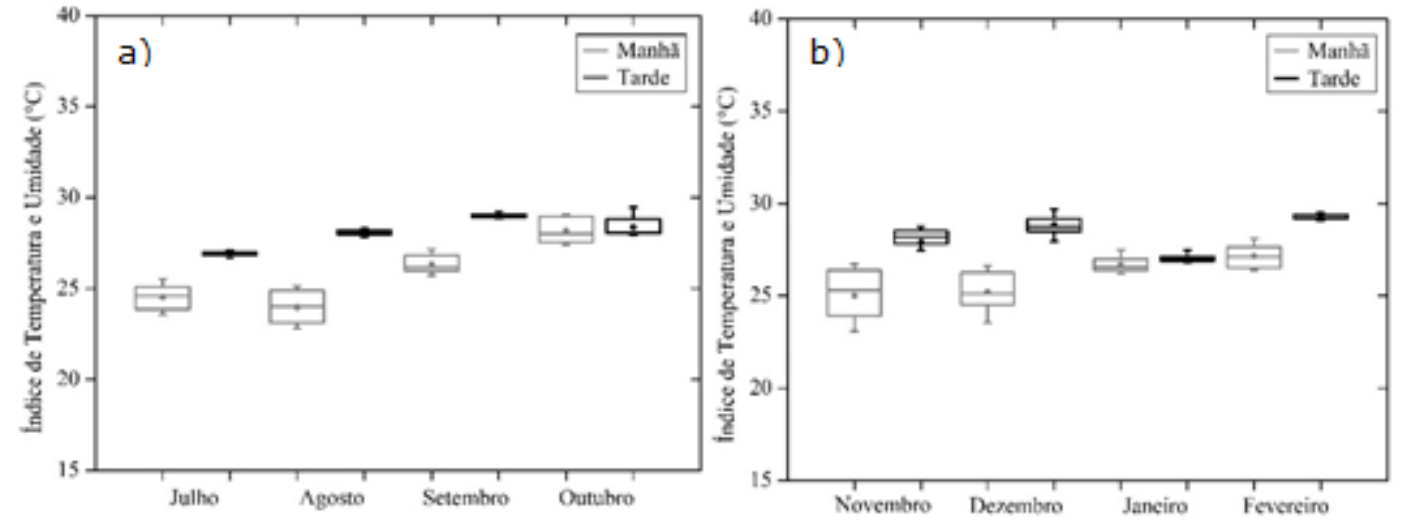

Figura 10 a) - Índice de Temperatura e Umidade no intervalo de estiagem b) -Índice de Temperatura e Umidade no intervalo chuvoso 
Semelhantemente aos $\left(\operatorname{ITU}^{\circ} \mathrm{C}\right)$ registrados para o parque, na estação fixa urbano os menores valores também foram aferidos no intervalo chuvoso e no período da manhã, quando comparado com o intervalo de estiagem e no período da tarde.

Para o intervalo chuvoso, os valores mínimos de $\left(\right.$ ITU $\left.^{\circ} \mathrm{C}\right)$ variaram de 22,8 a $27,4^{\circ} \mathrm{C}$ no período da manhã e de 23,1 a $26,4^{\circ} \mathrm{C}$ no período da tarde. Já os valores máximos de $\left(\mathrm{T}^{\circ} \mathrm{C}\right)$ variaram de 26,7 a $28,1^{\circ} \mathrm{C}$ e de 27,5 a $30,3^{\circ} \mathrm{C}$ no período manhã e da tarde respectivamente, variando a escala de percepção de pouco desconforto a desconforto.

Resultados semelhantes foram encontrados no trabalho de Querino 2017, realizado na cidade de Porto Velho, em que a autora concluiu que o intervalo de chuvas propiciou a diminuição dos valores dos índices, reduzindo a sensação de desconforto na região, uma vez que, as chuvas auxiliam no resfriamento da superfície terrestre.

Dessa maneira, em termos de conforto térmico, o transecto realizado no parque apresentou melhor percepção termohigrométrica quando comparado com a estação fixa em área urbana, pontuando os benefícios das áreas verdes.

\section{CONCLUSÕES}

O estudo em questão mostrou que a morfologia do parque analisado contribui para que as condições termohigrométricas interfiram positivamente no microclima quando comparado com a estação fixa em região urbana. Para a variável temperatura do ar, houve redução de até $5,2^{\circ} \mathrm{C}$, enquanto que para a umidade relativa do ar o aumento foi de até $10 \%$.

Em relação ao conforto térmico, o parque variou a escala de percepção de conforto a desconforto e a estação fixa em região urbana variou de pouco desconforto a desconforto. Dessa forma, os resultados sugerem que mesmo o parque alcançando melhores percepções, ainda se faz necessário a realização de estudos na área, na tentativa de aprimorar tais percepções, como por exemplo, a aplicação de questionários e o emprego de novos índices.

\section{REFERÊNCIAS}

AKBARI H., DAVIS S., DORSANO S., HUANG J., WINETT S. Cooling our communities - A guidebook on tree planting and light colored surfacing U.S. Environmental Protection Agency, Office of Policy Analysis, Climate Change Division, Washington D.C. (1997).

AKBARI H. \& LEVINSON R.. Evolução dos padrões de telhado frio nos EUA Advances in Building Energy Research , 2 (1) (2008), pp. 1 - 32.

ALGECIRAS J. A. R., CONSUEGRA L. G., MATZARAKIS A. Spatial-temporal study on the effects of urban street configurations on human thermal comfort in the world heritage city of Camagüey-Cuba. Building and Environment. 101 (2016), pp. 85-101.

AMERICAN SOCIETY FOR TESTING AND MATERIALS. Designation: D4430 - 00: Standard Practice for Determining the Operational Comparability of Meteorological Measurements, Estados Unidos da America, 2015. 
ARNFIELD J. A. Two decades of urban climate research: A review of turbulence, exchanges of energy and water, and the urban heat island. International Journal of Climatology, 23 (1) (2003), pp. 1-26.

ASSOCIAÇÃO BRASILEIRA DE NORMAS TÉCNICAS. NBR 17025/2017: Requisitos gerais para a competência de laboratórios de ensaio e calibração: Rio de Janeiro, 2017.

BARRADAS V. L. Air temperature and humidity and human comfort index of some city parks of Mexico city. International Journal of Biometeorology. 35 (1991), pp. 24-28.

BESIR A. B., CUCE E. Green roofs and facades: A comprehensive review. 82 (1) (2018), pp. 915-939.

CARTALIS C., SYNODINOU A., PROEDROU M., TSANGRASSOULIS A., SANTAMOURIS M. Modifications in energy demand in urban areas as a result of climate changes: An assessment for the southeast Mediterranean region Energy Conversion and Management, 42 (14) (2001), pp. 1647-1656.

CHU A., LIN Y., CHIUEH P. Incorporating the effect of urbanization in measuring climate adaptive capacity. Land Use Policy.(68) (2017), pp. 28-38.

DE PAULA D. C. J. Análise termohigrométrica pós intervenções urbana em Cuiabá-MT. Dissertação (Mestrado em Física Ambiental) - Instituto de Física da Universidade Federal de Mato Grosso, Cuiabá. (2017), pp. 1-90.

FERREIRA D.F. Análises estatísticas por meio do Sisvar para Windows versão 4.0. In...45a Reunião Anual da Região Brasileira da Sociedade internacional de Biometria. UFSCar, São Carlos-SP. (2000), pp. 255-258.

FRANCO F. M. Análise do comportamento termo-higrométrico urbano sob a ótica do uso e ocupação do solo em Cuiabá-MT. Tese (Doutorado em Física Ambiental) - Instituto de Física da Universidade Federal de Mato Grosso, Cuiabá. (2013), pp. 1-124.

FRANCO F. M., NOGUEIRA M. C. de J. A. Análise microclimática em função do uso e ocupação do sol em Cuiabá-MT. (Microclimatic analysis as function of land use in Cuiabá-MT). Mercator. 11 (26) (2012), pp. 157-170.

FRANCO F. M., NOGUEIRA M. C. de J. A., PINTO JÚNIOR O. B., BIUDES M. S., NOGUEIRA J. de S. Traçado urbano e sua influência no microclima: Um estudo de caso em centro histórico. (Urban tracing and its influence in the microclimate: A case study in historical center). Revista Eletrônica em Gestão, Educação e Tecnologia Ambiental. 9 (9) (2013), pp. 1916-1931.

INTERNATIONAL ORGANIZATION FOR STANDARDIZATION (ISO). ISO 7726: thermal environments: instruments and methods for measuring physical quantities. Switzerland, 1998.

KALESSI T., SANTAMOURIS M., SYNNEFA A., ASSIMAKOPOULOS D. DIDASKALOPOULOS P., APOSTOLAKIS K. Development and testing of PCM doped cool colored coatings to mitigate urban heat island and cool buildings Building and Environment. , 46 (3) (2011), pp. 570 - 576.

KOLOKOTRONI M., GIANNITSARIS I., WATKINS R. The effect of the London urban heat island on building summer cooling demand and night ventilation strategies Solar Energy, 80 (4) (2006), pp. 383-392. 
KOTHARKAR R., RAMESH A., BAGADE A. Urban Heat Island studies in South Asia: A critical review. Urban Climate. (2018), 16 pp.

LIMA, L. C. Conforto térmico em espaços abertos: Estudo de caso em um Parque Urbano na cidade de João Pessoa-PB. Dissertação (Mestrado em Engenharia Civil e Ambiental) - Centro de Tecnologia, Universidade Federal da Paraíba, João Pessoa. (2016), pp. 1-122.

LIN W., YU T., CHANG X., WU W., ZHANG Y. Calculating cooling extents of green parks using remote sensing: Method and test. Landscape and Urban Planning. 134 (2015), pp. 66-75.

MAHMOUD A.A.H. Analysis of the microclimatic and human comfort conditions in an urban park in hot and arid regions. Building and Environment. 46 (2011), pp. 2641-2656.

MAITELLI, G. T. Uma abordagem tridimensional de clima urbano em área tropical continental: o exemplo de Cuiabá-MT. Tese (Doutorado) Faculdade de Filosofia, Letras e Ciências Humanas, Universidade de São Paulo, São Paulo. (1994).

MAITELLI, G. T. Interações Atmosfera-Superfície. In: Moreno G.; Higa T. C. S. organizadores. Geografia de Mato Grosso: território, sociedade e ambiente. Entrelinhas: Cuiabá. (2005), pp. 1-296.

MARRA, N. C. S. N. Avaliação do conforto térmico em conjunto habitacional de interesse social-Simulação computacional com o programa Solene Microclima. (Mestrado em Ambiente Construído e Patrimônio Sustentável) - Escola de Arquitetura, Universidade Federal de Minas Gerais, Belo Horizonte. (2017), pp. 1-147.

MIHALAKAKOU G., SANTAMOURIS M., ASIMAKOPOULOS D. On the cooling potential of earth to air heat exchangers. Energy Conversion and Management. 35 (5) (1994), pp. 395 - 402.

MIHALAKAKOU G., SANTAMOURIS M., ASIMAKOPOULOS D. Modeling ambient air temperature time series using neural networks Journal of Geophysical Research D: Atmospheres, 103 (D16) (1998), pp. 19509-19517.

MIRZAEI P. A., HAGHIGHAT F. Approaches to study urban heat island - Abilities and limitations. Building and Environment, 45 (10) (2010), pp. 2192-2201.

NASTRAN M., KOBAL M., ELER K. Urban heat islands in relation to green land use in European cities. Urban Forestry \& Urban Greening. (2018), 9 pp.

NINCE P. C. do C. Vegetação e revestimentos urbanos: Implicações na sensação térmica dos usuários do campus da UFMT EM Cuiabá-MT. Tese (Doutorado em Física Ambiental) - Instituto de Física da Universidade Federal de Mato Grosso. (2013), pp. 1-90.

OKE T. R. City size and the urban heat island. Atmospheric Environment. 7 (8) (1973), pp. 769-779.

OKE T.R. The energetic basis of the urban heat island (Symons memorial lecture, 20 May 1980). Quarterly Journal of the Royal Meteorological Society, 108 (455) (1982), pp. 1-24. 
PETRALLI M. L., MASSETTI S., ORLANDINI. Air temperature distribution in an urban park: Differences between open-field and below a canopy The seventh international conference on urban climate, Yokohama, Japan (2009).

QUERINO, J. K. A. S. Caracterização termo higrométrica e de conforto térmico em porto velho no período de transição seco-chuvoso. (Doutorado em Física Ambiental) - Instituto de Física, Universidade Federal de Mato Grosso, Cuiabá. (2017), pp. 1-58.

ROSSI, S. A. Proposição de metodologia e de modelo preditivo para avaliação da sensação térmica em espaços abertos em Curitiba. (Doutorado em Tecnologia) Universidade Tecnológica Federal do Paraná, Curitiba. (2012), pp. 1-188.

SANTAMOURIS M., XIRAFI F., GAITANI N., SPANOU A., SALIARI M., VASSILAKOPOULOU K. Improving the microclimate in a dense urban area using experimental and theoretical techniques - The case of Marousi, Athens International Journal of Ventilation, 11 (1) (2012), pp. 1-16.

SANTOS F. M. de M. Clima urbano de Cuiabá-MT: ocupação do solo e suas influências. (Urban climate of Cuiabá-MT: Soil occupancy and its influences). Monografias ambinetais. 12 (12) (2013), pp. 2749-2763.

SANTOS F. M. de M. Influência da ocupação do solo na variação termohigrométrica na cidade de Cuiabá-MT (Doutorado em Física Ambiental) Instituto de Física da Universidade Federal de Mato Grosso, Cuiabá. (2012), pp. $1-88$.

SCHWARZ N., LAUTENBACH S., SEPPELT R. Exploring indicators for quantifying surface urban heat islands of European cities with MODIS land surface temperatures. Remote Sens. Environ. 115 (12) (2011), pp. 3175-3186.

SECRETARIA DE ESTADO DO MEIO AMBIENTE DE MATO GROSSO. Plano de Manejo do Parque Estadual Zé Bolo Flô. 2012;237 p.

SKOULIKA F., SANTAMOURIS M., KOLOKOTSA D., BOEMI N. On the thermal characteristics and the mitigation potential of a medium size urban park in Athens, Greece. Landscape and Urban Planning. 123 (2014), pp. 73-86.

SUN S., XU X., LAO Z., LIU W., LI Z., GARCIA EH., ELE L., ZHU J. Evaluating the impact of urban green space and landscape design parameters on thermal comfort in hot summer by numerical simulation. Building and Environment. 123 (2017), pp. 277-288.

THOM E. C. The discomfort index. Weatherwise, Boston. 12 (1) (1959) pp. 5760.

TONG S., WONG N. H., TAN C. L., JUSUF S. K., IGNATIUS M., TAN E. Impact of urban morphology on microclimate and thermal comfort in northern China. Solar Energy. 155 (2017), pp. 212-223.

UNITED NATIONS. World Urbanization Prospects. Department of Economic and Social Affairs, New York. 2014; 27 pp.

VALIN JUNIOR M. de O., RIBEIRO K. A., SANTOS F. M. de M., NOGUEIRA M. C. de J. Análise da variação higrotérmica entre área rural e urbana em período de nível crítico de umidade no Vale do Rio Cuiabá. Revista Eletrônica em Gestão, Educação e Tecnologia Ambiental. 19 (2015), pp. 1392-1399. 
VALIN JR, M. O.; SANTOS, F. M. M.; RIBEIRO, K. F. A.; NOGUEIRA, M. C. J. A.; NOGUEIRA, J. S. Análise da relação entre abrigos meteorológicos alternativos para pontos fixos e o comportamento de variáveis termo-higrométricas. Revista Brasileira de Climatologia. 18 (2016), pp. 157-179.

VAREJÃO M. A. S. Meteorologia e Climatologia. Versão Digital. Recife, Pernanbuco - Brasil. (2006).

XAMAN J., CISNEROS-CARREÑO J., HERNÁNDEZ-PÉREZ I., HERNÁNDEZ-LOPEZ I., AGUILAR-CASTRO K. M., MACIAS-MELO E. V. Thermal perfomance of a hollow block with/without insulating and reflective materials for roofing in Mexico. Applied Thermal Engineering. 123 (2017), pp. 243-255.

XAVieR A. L., NOGUeiRA M. C. de J. A., MAITELli G. T., OliVeirA A. G., OLIVEIRA A. S., SANTOS F. M. de M., NOGUEIRA J. S. Variação da temperatura e umidade entre áreas urbanas de Cuiabá (Variation of the temperature and humidity among urban areas of Cuiabá). Engenharia Ambiental. 6 (1) (2009) pp. 082-093.

ZHANG L. MENG X., LIU F., XU L., LONG E. Effect of retro-reflective materials on temperature environment in tents. Case Studies in Thermal Engineering. 9 (2017), pp. 122-127.

ZHANG Y., ZHANG L., PAN Z., MENG Q., FENG Y., CHEN Y. Hydrological properties and solar evapirative cooling performance of porous clay tiles. Construction and Building Materials. 151 (2017), pp. 9-17. 